gratulated on also discussing in a final chapter the eugenic implications of genetic counselling. The effects of mutation rate and altered fitness for unilocal conditions are set out in several Tables (there appear to be inconsistencies in some of these which will need correction in a later edition). The authors note that the entire impact of fresh recessive mutation can be borne by a mild reduction in the planned fitness of heterozygotes. They might have made the further point that, in the future, it will probably not be the practice to select against individual recessives, but to evaluate the total number of recessives that an individual couple carry between them.

On positive eugenics the authors rightly stress the difficulty of knowing just which qualities will be socially desirable several generations hence. They also stress that, in a democratic society, individual parents have to be judges in their own case and themselves make the decision about the likely genetic worth of their children. Many would take the view that such decisions are, nevertheless, a moral responsibility that every couple must shoulder.

Cedric CARTER

Experimental Biology and Medicine Cytogenetic aspects of Malignant Transformation

By N. B. Atkin. Vol. 6. Series Editors: A., Wolsky, D. J. Pizzarella, G. V. Sherbet, J. Steiner. (Pp. viii + 168; 21 Figures + 9 Tables. DM. 74.) Basel: $S$. Karger. 1976.

The author has provided, within the confines of this short monograph, a comprehensive and reasonably up-to-date review of many aspects of chromosomes and malignancy.

The early chapters survey the kinds of chromosome abnormalities found in malignant cells, chromosome breaking agents, and constitutional chromosome abnormalities (including the 'breakage syndromes') which may predispose to cancer, and there is a brief consideration of premalignancy. The greater part of the text is taken up with details of the chromosome changes found in the leukaemias and various solid tumours of man. There are then short chapters dealing with tumours of other animals and plants, various in vitro methods for studying malignancy, and some general conclusions. The book is by no means profusely illustrated, but the figures are generally adequate and well chosen.

Dr Atkin describes his book as a progress report and it is unfortunate that, even now, little cytogenetic information is available from some tumour types, which is not of prebanding vintage. This is particularly true of many solid tumours, where technical difficulties in obtaining good cell suspensions and $\mathbb{\Phi}$ adequate numbers of mitoses mean that it will be some $\mathbb{\Phi}$ time before much systematically collected banding data become available. On the other hand, bone $\stackrel{?}{\longrightarrow}$ marrow is a very suitable tissue for chromosome studies, and consequently a great deal has been learnt $\underline{\underline{E}}$ recently of the precise cytogenetic changes which take $\frac{\bar{\sigma}}{\frac{5}{5}}$ place in both malignant and premalignant haemato- $\stackrel{\mathbb{}}{\propto}$ logical disorders: much of this information may be found here (there are references cited up to the middle ${ }_{\vec{D}}$ of 1975).

The main shortcoming of this monograph is that it $\overrightarrow{\vec{A}}$ is very largely descriptive. However, with nearly $800 \stackrel{\mathrm{N}}{\mathrm{W}}$ references, it may be recommended as a valuable $\overline{3}$ background for all workers in the field of cancer cyto- $\frac{0}{-}$ genetics.

B. R. REEVES:-

\section{Chemical Mutagens. Principles and Methods for their Detection}

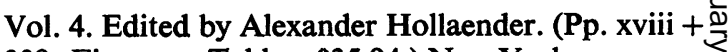
339; Figures + Tables. \$35.94.) New York:

Plenum Press. 1976.

Recent years have seen a massive upsurge of interest in the potential genetic hazard to man of environ- $O$ mental chemicals. This has been reflected in the first three volumes of Hollaender's series Chemical Mutagens. The latest addition is not for those who $\frac{\not}{\not}$ seek an introduction to or a justification for genetic $\supseteq$ toxicology - earlier volumes will suffice for that-but $\overrightarrow{\overrightarrow{0}}$ it contains much of value and relevance to those 3 interested in environment or industrial medicine. The past two years have seen strong support for the? somatic mutation theory of cancer or, to be more precise, for a strong correlation between carcinogenicity and the ability of an agent to damage the hereditary material DNA. This correlation is accepted. by most of the contributors to Volume 4 excepto Lijinsky who, in a characteristically idiosyncratic chapter on N-nitroso compounds, nevertheless음 manages to be both informative and challenging. $>$ Though he might not accept this, we probably do now? know where to look for answers to the problems of $N$ chemical carcinogenicity raised by Lijinsky such as. organ and species specificity, even if we do not yet know what they are.

Medicines are, almost by definition, biologicallyo active substances and it is not surprising that a numbero of them turn out to be mutagenic, usually after metabolic activation occurring within the body. Legator, $?$ Zimmering, and Connor contribute a useful chapter on ways of detecting mutagenic activity in the blood웅 or urine of exposed animals and patients. Many? 